Article

\title{
Optimizing Spacer Length for Positioning Functional Groups in Bio-Waste
}

\author{
Revathi Iyengar *, Maria Faure-Betancourt, Saleh Talukdar, Jinting Ye and Abel E. Navarro \\ Science Department, Borough of Manhattan Community College, The City University of New York, New York, \\ NY 10007, USA; mariaalejandr.faure-betancourt@jjay.cuny.edu (M.F.-B); saleh.talukdar@stu.bmcc.cuny.edu (S.T.); \\ jinting.ye@stu.bmcc.cuny.edu (J.Y.); anavarro@bmcc.cuny.edu (A.E.N.) \\ * Correspondence: riyengar@bmcc.cuny.edu; Tel.: +1-212-220-8000
}

Received: 8 August 2018; Accepted: 30 August 2018; Published: 2 September 2018

\begin{abstract}
The goal of this study was to determine the optimal chain length needed for tethering functional groups on bio-wastes. The purpose of modifying the surface of bio-waste is to improve their affinity for phenols. To this end, four different aminated green tea leaves, with the amine group located at the end of $6,8,10$, and 12 carbons were synthesized. Green approaches to functionalization lead to fewer reactive sites. Optimizing spacer length is one way to ameliorate this. The aminated tea leaves were prepared by a tosylation reaction followed by displacement with a diamine used in excess. The tea leaves with the amine at the end of six carbons proved to have the best ability to remove 2-chlorophenol (2-CP) from its aqueous solution. It was at least 3-4 times better than native spent tea leaves. The mechanism by which the phenol was removed proved to be primarily an acid-base reaction followed by $\mathrm{H}$-bonding and dipole-dipole interactions. Because of the acid-base interactions, the relatively low-boiling 2-CP did not volatilize off the aminated tea leaves enabling recycling. On the other hand, with activated charcoal, the adsorbed 2-CP volatilized almost completely under ambient conditions.
\end{abstract}

Keywords: chain length; spacer; tethering; phenol; bio-waste; 4-aminoantipyrine; spent tea leaves; tosylation and amination

\section{Introduction}

Prospects in water treatment keep booming as freshwater availability declines. Phenolics constitute about $10 \%$ of the priority pollutant list for water issued by the EPA (Environmental Protection Agency, Washington, DC, USA). Activated carbon is a great adsorbent of organics. However, it needs to be heated for it to be in the active state required for adsorption [1]. Biomass is known to bind contaminants onto its cellular structure by a physicochemical process referred to as biosorption [2-4]. Bio-wastes, such as spent tea leaves, are largely untapped materials to develop inexpensive and effective tools for wastewater treatment.

Phenols, being acidic in nature, need sites on the bio-waste to be basic enough for efficient removal. However, unmodified bio-wastes do not have enough of these strongly basic sites needed for phenol removal.

Modifying surfaces to improve reactivity is a known idea implemented widely on artificial polymers [5]. The challenge with modifying a bio-based material is that the main functional group available for performing any reactions is the hydroxy group. The approach of modifying lignocellulosic material is currently explored by combining mechanical and chemical approaches, with a particular emphasis on green aspects [6,7]. The effort so far has been geared towards creating carboxyl groups. Carboxyl groups, being acidic in nature, are not expected to interact with the acidic phenolic group. Rather, a basic group, such as an amine, can remove the phenol by an acid-base reaction. 
Modification of lignocellulosic material is difficult due to the complexity of the bio-waste. For example, "spent" green tea leaves contain about 20 weight percent of phenolics and saponins and 25 weight percent of crude proteins [8] in addition to the cellulose. In a preliminary work, aminated and thiolated leaves were prepared following the procedure developed by Abel et al. [9]. These modified tea leaves exhibited significant adsorption of $\mathrm{Cu}(\mathrm{II})$ ions in aqueous systems compared to the unmodified "spent" tea leaves (Table 1).

Table 1. Comparison of $\mathrm{Cu}$ (II) removal by modified tea leaves.

\begin{tabular}{cccc}
\hline Name, Group & Chain Length & $\begin{array}{c}\text { Adsorption (\%) } \\
\text { (Unmodified) }\end{array}$ & $\begin{array}{c}\text { Adsorption (\%) } \\
\text { (Modified) }\end{array}$ \\
\hline Thiol, $-\mathrm{SH}$ & eight (six methylenes and two oxygens) & 29 & 49 \\
Amine, $-\mathrm{NH}_{2}$ & six methylenes & 27 & 47 \\
\hline
\end{tabular}

One reason for the enhanced interaction between the newly introduced functional group and the Copper (II) ions is thought to be due to the chain length onto which the group is tethered. This has been suggested even in the work of Abel et al. [9], where the maximum benefit for their application (anti-bacterial activity) was observed when the chain length was 16 methylenes long. Amines are known to react with phenols forming the corresponding ammonium salts. Therefore, the idea was to identify the optimal "spacer" for amines.

For this study, the optimum chain length was determined based on the effectiveness as a 2-CP absorbent. There is considerable literature evidence that functional groups linked directly to a glucose ring without a spacer impose considerable steric hindrance. For example, if the thiol group is linked directly to the glucose ring, the abstraction of hydrogen from the SH by a polymeric radical [10] is inhibited.

The goal of this project is to develop eco-friendly filters using raw solid wastes to effectively remove phenols as part of a water treatment process. Another advantage would be the easy regeneration of the solid waste with a base to obtain the phenols back for reuse in other manufacturing processes.

\section{Materials and Methods}

The synthetic scheme using hexamethylene diamine for placing the amino group at the end of a six-carbon chain is shown in Scheme 1. The same scheme was used with the following amines: 1,8-diaminooctane, 1,10-diaminodecane, and 1,12-diaminododecane. This tethered the amine groups on the spent green tea leaves with varying chain lengths. This scheme extends work done by Abel et al. to modify cotton [9].

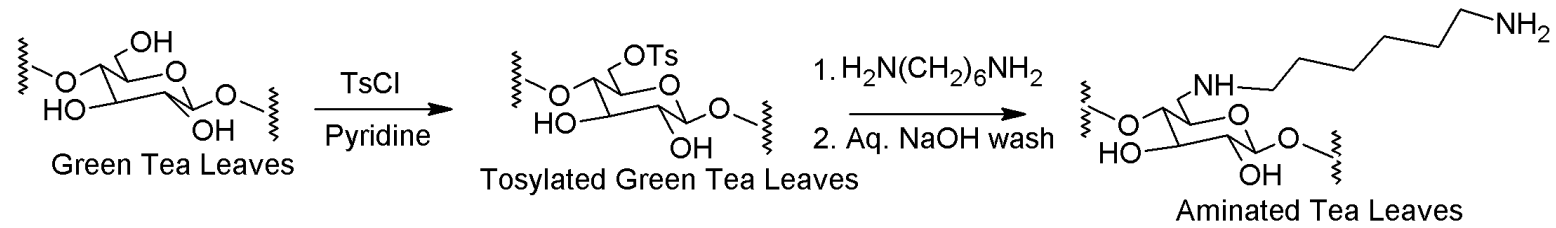

Scheme 1. Synthetic scheme for tethering the amine functionality.

\subsection{Solvents and Reagents}

The water used in all experiments was the Millipore filtered de-ionized (DI) water. Solvents, such as pyridine and acetonitrile, were used as supplied without further purification in the synthetic steps. Pyridine was purchased from Acros Organics (Pittsburgh, PA, USA). ACS Certified acetonitrile was obtained from Fisher Scientific (Pittsburgh, PA, USA). Pyridine and acetonitrile were used in the tosylation and amination steps, respectively. ACS Certified analytical grade ammonium chloride was 
obtained from Acros Organics (Pittsburgh, PA, USA). Ammonium hydroxide was obtained from Fisher Scientific (Pittsburgh, PA, USA). The 4-Aminoantipyrine was purchased from Sigma Aldrich (Atlanta, GA, USA) and used without any further purification. Potassium ferricyanide (for analysis, $99+\%$ ) was obtained from Acros Organics (Pittsburgh, PA, USA). ACS Grade 2-Chlorophenol from Acros Organics (Pittsburgh, PA, USA) was used in the studies. Solutions of 2-CP were always freshly prepared before use. Due to the photosensitive nature of 2-CP solutions, volumetric flasks containing its solution were always wrapped in aluminum foil. Amber colored glass tubes were used for equilibration studies to protect the 2-CP from any decomposition. Activated carbon (AC), NORIT ${ }^{\mathrm{TM}}$ SA 2, from Acros Organics (Pittsburgh, PA, USA) was used in the study.

The biowaste used for the project was obtained from green tea bags. The bags were boiled in water repeatedly to remove all water-soluble compounds. The tea bags were then dried in the oven. The spent tea leaves were removed from the bags and subjected to Soxhlet extraction with a 1:1 mixture of ethanol and acetone ( $400 \mathrm{~mL}$ of solvent mixture per $20 \mathrm{~g}$ of spent tea leaves). The tea leaves were dried in the oven at $50{ }^{\circ} \mathrm{C}$ till constant weight. Soxhlet extracted green tea leaves are abbreviated as SEGT.

\subsection{Preparation of Tosylated Green Tea (TosGT)}

$\mathrm{TsCl}(253.8 \mathrm{~g}, 1.3 \mathrm{~mol})$ was placed in a $1 \mathrm{~L}$ Wheaton bottle. Soxhlet Extracted Green Tea (SEGT) ( $31.5 \mathrm{~g}, 0.194 \mathrm{~mol}$ ) was added to the tosyl chloride. For the calculations, SEGT was assumed to be $100 \%$ cellulose, and only one $-\mathrm{OH}$ group from an anhydroglucose unit (AGU unit, molecular weight (MW) $=162 \mathrm{~g} / \mathrm{mol})$ was considered to react. Pyridine $(600 \mathrm{~mL})$ was added to the bottle and sonicated for $20 \mathrm{~min}$ followed by magnetic stirring at room temperature for $96 \mathrm{~h}$. The reason for a reaction time of $96 \mathrm{~h}$ can be found under results and discussion. The pyridine solution was then decanted off into a waste container. Ice cold water was added to dissolve the pyridine clinging to the surface of the tea leaves [11]. The tea leaves were washed five times with ice-cold water ( 200 $\mathrm{mL}$ each time) to remove pyridine and other by-products. The washes were monitored using $\mathrm{pH}$ paper till neutrality. The tea leaves were then washed with acetone thoroughly. The purpose of the acetone washes was to remove un-reacted tosyl chloride. The acetone washes were monitored using a silica gel plate impregnated with a fluorescent material and UV lamp. The tea leaves were then vacuum-filtered. They were air dried to constant weight.

The tosylation step produced $40.91 \mathrm{~g}$ of the product from $31.48 \mathrm{~g}$ of SEGT (Soxhlet Extracted Green Tea). Its purity was estimated to be at least $97 \%$, based on the amount of chlorine present in the tosylated tea leaves, as determined by elemental analysis. The number of moles of tosyl group per gram of the tosylated material was estimated based on the increase of mass after the tosylation step. This was found to be $1.5 \mathrm{mmol} / \mathrm{g}$ of TosGT.

\subsection{Preparation of the Various Aminated Green Teas (C6-AGT; C8-AGT; C10-AGT; and C12-AGT)}

The tosylated tea leaves were subjected to amination using four different diamines to produce four different kinds of aminated tea leaves. The diamines used were hexamethylene diamine (C-6 diamine), 1,8-octanediamine (C-8 diamine), 1,10-decanediamine (C-10 diamine), and 1,12-dodecanediamine (C12-diamine). This resulted in the displacement of the tosyl group by one of the amino groups in the diamine while the second amine group remained attached to the other end. This resulted in a family of aminated tea leaves with the amine functionality tethered at the end of varying chain lengths. Cross-linking was prevented by targeting a 1:8 mole ratio of tosylate functionality to the diamine.

Each diamine $(0.12 \mathrm{~mol})$ was placed in a $500 \mathrm{~mL}$ bottle and dissolved in acetonitrile $(250 \mathrm{~mL})$. The C-10 diamine (1,10-decanediamine) and C-12 diamine (1,12-dodecanediamine) were distinctly less soluble than the C-8 diamine (1,8-octanediamine) and the C- 6 diamine (hexamethylenediamine). Tosylated tea leaves $(10 \mathrm{~g})$ were then poured into the acetonitrile solution containing the varying diamines. The mixture was initially sonicated for $20 \mathrm{~min}$ followed by magnetic stirring at room temperature ( $19 \mathrm{~h}$ in the case of the $\mathrm{C}-8$ diamine; $68 \mathrm{~h}$ for the other three diamines). The acetonitrile 
solutions containing the diamine were decanted off at that point. Ice cold aqueous $\mathrm{NaOH}$ solution $(2 \mathrm{~N}, 500 \mathrm{~mL}$ ) was added followed by magnetic stirring ( $5 \mathrm{~min}$ ). Stirring with the base solution was carried out to accomplish the conversion of the ammonium tosylate salt to the diamine as shown in Scheme 2. Note that Scheme 1 does not show the ammonium tosylate formation explicitly.

The tea leaves were transferred to a beaker and distilled water $(200 \mathrm{~mL})$ was added to it. Five water washes were performed to remove all traces of sodium hydroxide followed by acetone washing to remove traces of diamines. This was monitored by spotting the acetone washes on a wet $\mathrm{pH}$ paper. The tea leaves were then vacuum filtered. In the case of the C-12 diamine, ethanol washes were used initially before the acetone washes to aid in its complete removal. The four aminated tea leaves (each weighing in the range of 8.7-9.2 g) were air dried to constant weight and bottled off till further use.

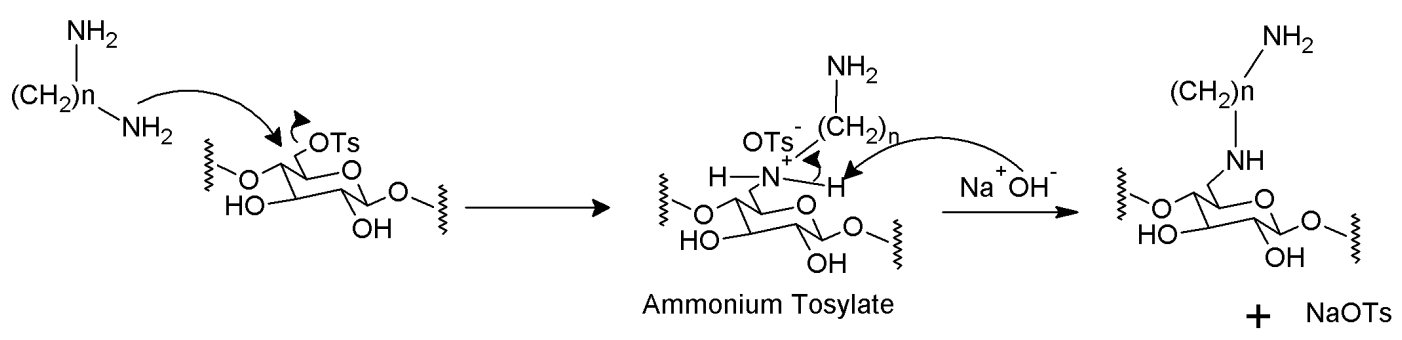

Scheme 2. Conversion of ammonium tosylate to the secondary amine.

\subsection{Characterization}

Elemental Analyses were performed at the Robertson Microlit Laboratories in Ledgewood, New Jersey. A Frontier Fourier transform infrared (FTIR) spectrometer (Perkin Elmer, Waltham, MA, USA) with a Universal Attenuated Total Reflectance (UATR) attachment was used for obtaining infrared spectra of the chemically modified tea leaves. Pictures of the morphological texture were obtained using a tabletop scanning electron microscope, Model TM3000, from Hitachi High-Technologies (Pleasanton, CA, USA). Samples were viewed directly without gold coating. Quantax 70, an optical accessory for TM 3000, was used to obtain energy dispersive X-ray spectra (EDS). It was purchased from Bruker (Billerica, MA, USA). This was used to gauge the relative abundance of elements on the surface of chemically modified tea leaves.

\subsection{Equilibrium Conditions for Adsorption}

Amber glass tubes $(8 \mathrm{~cm}$ long with a diameter of $2.5 \mathrm{~cm}$ ) with Teflon-lined screw caps were used for the equilibration experiments. Varying amounts of the aminated tea leaves and solutions containing 2-CP at the appropriate concentrations were placed in these tubes. Targeted concentrations of 2-CP solutions were prepared by making a stock solution of $1000 \mathrm{ppm}$ and diluting it with Millipore filtered DI water to the desired concentration.

The tubes were placed in the "built-in" sample holders of the incubator/shaker. The I24 incubator shaker used in the study was purchased from New Brunswick Scientific (Edison, NJ, USA). Equilibration was performed at ambient conditions overnight at a setting of $250 \mathrm{rpm}$ (revolutions per minute). Agitation involved horizontal circular motions.

\subsection{Analysis: Phenol Estimation}

This was based on the spectrophotometric method described in the 21st edition of "Standard Methods for the examination of Water and Wastewater" [12]. According to the procedure, the sample, blank, and standards should all be at a $\mathrm{pH}$ of $10 \pm 0.1$ with the buffer used.

The microplate reader was purchased from Molecular Devices (San Jose, CA, USA). Absorbance measurements at $500 \mathrm{~nm}$ were obtained using SpectraMax iD3 from Molecular Devices (San Jose, CA, USA). A UV-transparent microplate with 96 wells (well capacity of $500 \mu \mathrm{L}$ ) was used for performing 
the absorbance measurements at $500 \mathrm{~nm}$. The absorbance of the blank plate was initially obtained. The blank absorbance values of the respective wells were subtracted from the observed values with sample solutions.

The 4-aminoantipyrine method [12] was adapted to be performed in Eppendorf vials (1.5 mL) as follows: to $910 \mu \mathrm{L}$ of the buffer solution, $50 \mu \mathrm{L}$ of the phenol solution and $20 \mu \mathrm{L}$ each of the potassium ferricyanide and 4-aminoantipyrine solutions were added. After mixing, $200 \mu \mathrm{L}$ of the mixture was used for plating the wells. Absorbance measurements were made within 15 min of combining the solutions.

Analyses were performed in duplicate. The original phenol solutions used in the different studies were analyzed. Amount of decrease in absorbance as compared to the absorbance of the original solution is reported as a percentage. The reported percentage amount of 2-CP removed is the mean value for the two analyses. Mean absolute deviation is also included.

\subsection{Desorption Studies}

Details of the adsorption/desorption pair of experiments are given in this section.

\subsubsection{Details of Adsorption}

C6-AGT $(0.5 \mathrm{~g})$ and activated carbon $(0.5 \mathrm{~g})$ were each placed in two different $250 \mathrm{~mL}$ Wheaton bottles and mixed with a 2-CP solution (100 mL, $75 \mathrm{ppm})$. The bottles were wrapped in aluminum foil. They were placed in the shaker and allowed to equilibrate at $250 \mathrm{rpm}$ overnight. Then, suspensions were gravity filtered and poured back in the bottles. This was repeated five times to ensure complete transfer of the solids on to the filter paper. They were allowed to air dry on the lab bench overnight. They had dried to constant weight at that point. The filtrate was analyzed to determine the percentage of 2-CP adsorbed.

\subsubsection{Details of Desorption}

One hundred and fifty milligrams (150 mg) of C6-AGT and activated charcoal that had adsorbed the 2-CP (see Section 2.7.1) were each placed in three of the amber tubes. The three tubes were filled with aqueous hydrochloric acid $(0.1 \mathrm{~N}, 30 \mathrm{~mL})$, the sodium hydroxide solution $(0.1 \mathrm{~N}, 30 \mathrm{~mL})$, and Millipore filtered DI (de-ionized) water $(30 \mathrm{~mL})$. The solutions were placed in the shaker and equilibrated at room temperature at a speed of $250 \mathrm{rpm}$ for $2 \mathrm{~h}$. The solutions were then analyzed for 2-CP following the procedure described in Section 2.6 for Phenol Analysis.

\section{Results and Discussion}

\subsection{Synthesis}

\subsubsection{Tosylation Step}

In the paper by Abel et al. [9], the tosylation was performed on cotton cloth, which is mostly cellulose. To determine the time needed for the reaction with SEGT, which has lignins and proteins in addition to the cellulose, an aliquot was removed at the $2 \mathrm{~h}$ period. At $2 \mathrm{~h}$, the percent sulfur, an indicator of tosyl groups incorporated in the tea leaves, based on elemental analysis, was found to be $1.9 \%$. That number rose to $2.7 \%$ when the reaction was allowed to proceed for $89 \mathrm{~h}$. According to Abel et al. [9], the primary hydroxyl site in the glucose ring was expected to be functionalized quite specifically relative to secondary sites within the same molecule. The authors presumed the tosylation to occur at the 6-position of the carbohydrate unit without any reaction at the available 2and 3-position hydroxyl sites.

However, as can be seen in the discussion in Section 3.1.2, the secondary hydroxyls appear to be tosylated. It is attributed to the extended reaction time employed for this step. Note that the molar excess used in the case SEGT was identical to that used in the case of cotton [9]. The percent sulfur 
jumped to $5.8 \%$ when the reaction was scaled up from $1 \mathrm{~g}$ to $30 \mathrm{~g}$. The only difference is that the reaction was allowed to progress a little further; a reaction time of 96 versus $89 \mathrm{~h}$. The other pertinent change during scaleup was the use of very dry SEGT as the starting material. It is therefore important to oven dry the soxhlet extracted green tea prior to performing the tosylation step.

The water-holding capacity of green tea leaves is approximately 6\% [8]. Thus, SEGT gained significant weight under ambient humid conditions when left out on the lab bench. It is therefore important to not only to dry the SEGT but also to store it in an airtight bottle until future use.

According to Abel et.al. [9], tosylation on cloth could be accomplished without the use of pyridine. Such tosylations in their case had been performed in acetonitrile medium with solid sodium carbonate present as an acid sink. The resultant modified carbohydrate materials exhibited the same anti-bacterial activity as those produced using pyridine as a base. In the case of tosylation reactions performed on the tea leaves, there was a significant difference in rates of reaction. Pyridine increased the rate of reaction significantly. For example, when tosylation was performed on a $1 \mathrm{~g}$ scale using $20 \mathrm{~mL}$ acetonitrile and $2 \mathrm{~g}$ sodium carbonate and the same number of equivalents of $\mathrm{TsCl}$ as in the case of using pyridine as the solvent, the percent sulfur was only 1.8 even after $24 \mathrm{~h}$ at room temperature. The reaction with pyridine as the solvent achieves this stage within $2 \mathrm{~h}$.

Using the sodium carbonate along with acetonitrile as the solvent also created a different challenge. Removing the sodium carbonate using water resulted in basic solutions that appeared to hydrolyze the tosylate group. The sodium carbonate hardened and formed a cake in which the tea leaves were trapped. Thus, it took longer for the leaves to be mechanically freed of the tea leaves causing the hydrolysis to occur.

\subsubsection{The Basis for the 1:8 Mole Ratio of Diamine to Tosylate}

The main challenge in the synthesis was to avoid cross-linking. The tosylate group in the polymeric backbone can be displaced by each of the amine groups; one at each end of the diamine. Thus, a cross-linked structure could result. Scheme 3 shows this using structural drawing where the jagged lines represent the polymer backbone.

The 1:8 mole ratio was based on the author's (RI) prior industrial experience. This prior work involved trans-esterification of a diol. Less than 6 weight percent diester was obtained when a 6-fold molar excess of the diol was used.

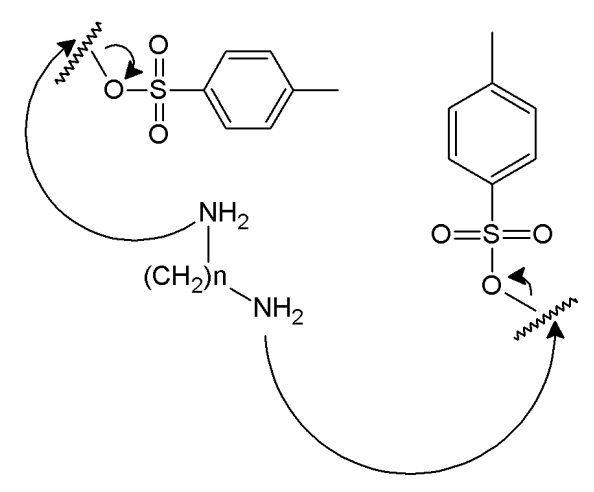

Scheme 3. Possible Cross-linking Mechanism.

\subsection{Characterization of SEGT, TosGT, and AGT Leaves}

\subsubsection{Elemental Analysis}

Elemental analysis was performed in duplicate. The average of the two analyses is summarized in Table 2. Note that the percentage of nitrogen in SEGT corroborates well with the suggestion of $25 \%$ crude protein in green tea leaves by Ramdani et al. [8]. As expected, in the case of TosGT, the percentage of sulfur increases dramatically and the percentage of nitrogen goes down. 
Since the chlorine analysis was not performed in duplicate, the surface of the tosylated green tea (TosGT) was analyzed using energy dispersive X-rays. As can be seen in Figure 1, the surface contains very little chlorine suggesting very little contribution, if any, from $\mathrm{TsCl}$ to the sulfur values seen in Table 2.

p-toluenesulfonyl chloride can hydrolyze to p-toluenesulfonic acid ( $\mathrm{p}$ - $\mathrm{TsOH})$. However, $\mathrm{p}$ - $\mathrm{TsOH}$ is extremely water soluble and has most likely been removed by multiple water washes as described in the experimental section. To determine whether the soxhlet extracted green tea used for the tosylation reaction contained chlorine, EDS of the SEGT was obtained. As can be seen from Figure 2, no detectable amount of chlorine was found in the starting sample.

In Table 2, the general trend noted with the aminated green tea leaves (AGT) is that the percent nitrogen increases and the percent sulfur decreases when compared to the TosGT. Using the percent sulfur in the various aminated tea leaves, the density of amine groups was estimated to be $0.4-0.6 \mathrm{mmol} / \mathrm{g}$. Interestingly, the tosylate group does not appear to be completely replaced by the amine. The most likely explanation for the tosylate not being fully displaced is the presence of secondary tosylate groups [13]. A displacement reaction at a secondary carbon would require higher temperatures due to the higher energy of activation associated with the transition state involved. Thus, the secondary tosyl groups, most likely, did not get displaced at ambient conditions. The displacement even of the primary tosyl groups might not have occurred to completion. However, using higher reaction temperatures was not considered in the interest of maintaining the structural integrity of the tea leaves.

Table 2. Elemental analyses.

\begin{tabular}{cccccc}
\hline Material & \% Carbon & \% Hydrogen & \% Nitrogen & \% Sulfur & \% Chlorine \\
\hline SEGT & 45.82 & 6.25 & 4.09 & 0.085 & $* * *$ \\
TosGT & 49.82 & 5.68 & 3.38 & 5.81 & $0.45^{*}$ \\
C6-AGT & 50.3 & 6.10 & 4.7 & 3.5 & $* * *$ \\
C8-AGT & 50.35 & 6.47 & 4.07 & 4.54 & $* * *$ \\
C10-AGT & 50.37 & 6.49 & 4.91 & 3.92 & $* * *$ \\
C12-AGT ** & 51.5 & 6.48 & 4.65 & 3.81 & $* * *$ \\
\hline
\end{tabular}

* Chlorine number was not obtained in duplicate. ${ }^{* *}$ These numbers are the average of a triplicate because of sample variations. ${ }^{* * *}$ Not obtained. SEGT, Soxhlet Extracted Green Tea; AGT, aminated green tea; TosGT, tosylated green tea.

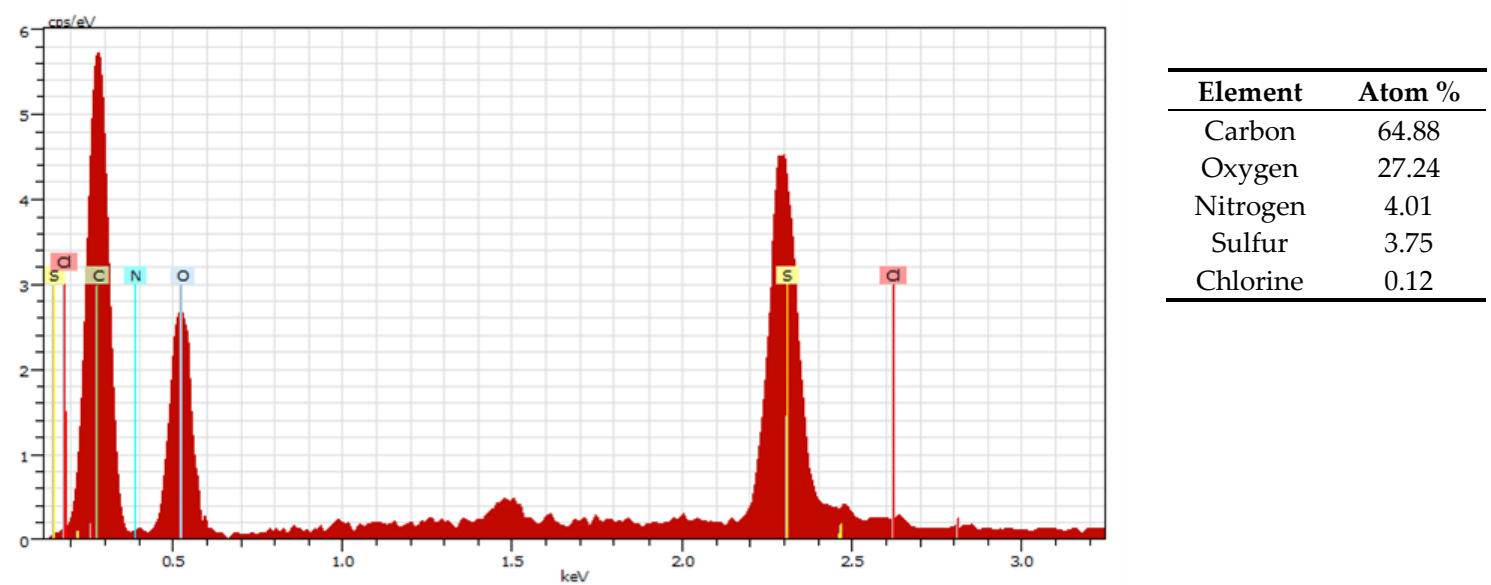

Figure 1. Energy Dispersive X-ray spectra and Atom Percent of the tosylated green tea (TosGT). 


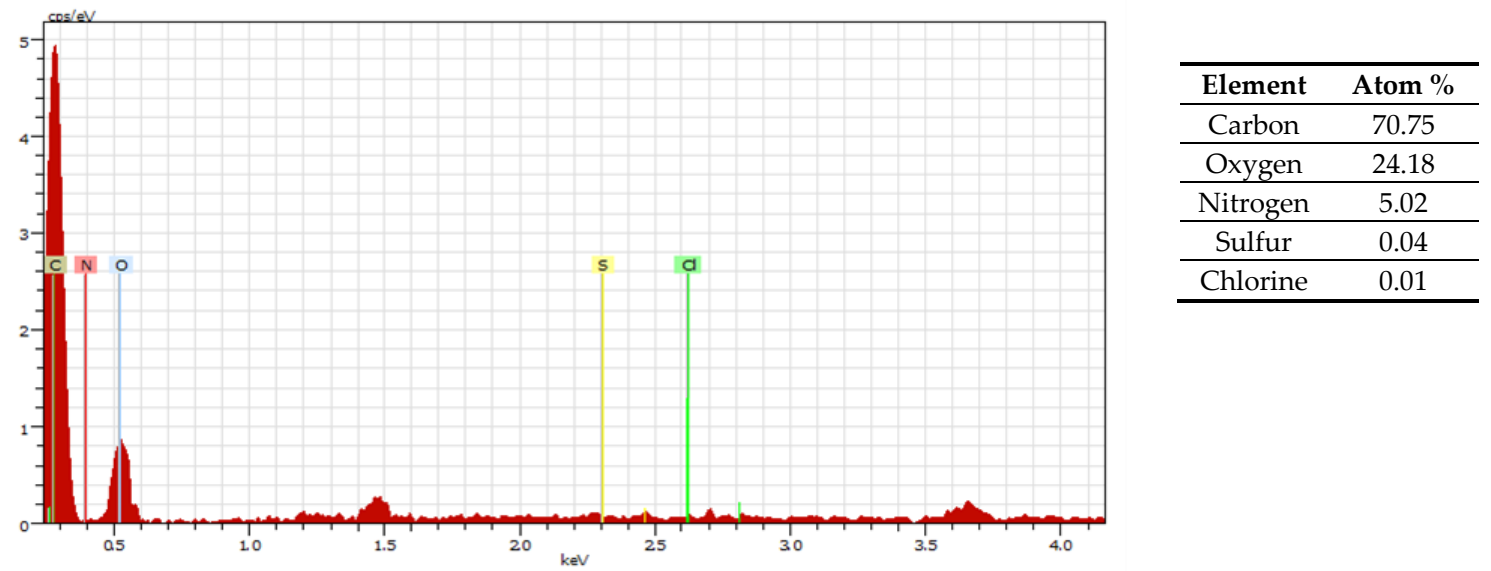

Figure 2. Energy Dispersive X-ray spectra and Atom Percent of SEGT.

EDS spectra of the aminated tea leaves were obtained to determine whether there were significant amounts of $\mathrm{N}$ on the surface of the aminated tea leaves. A typical spectrum is shown in Figure 3. As expected, the third most abundant element on the surfaces of the aminated tea leaves is nitrogen.

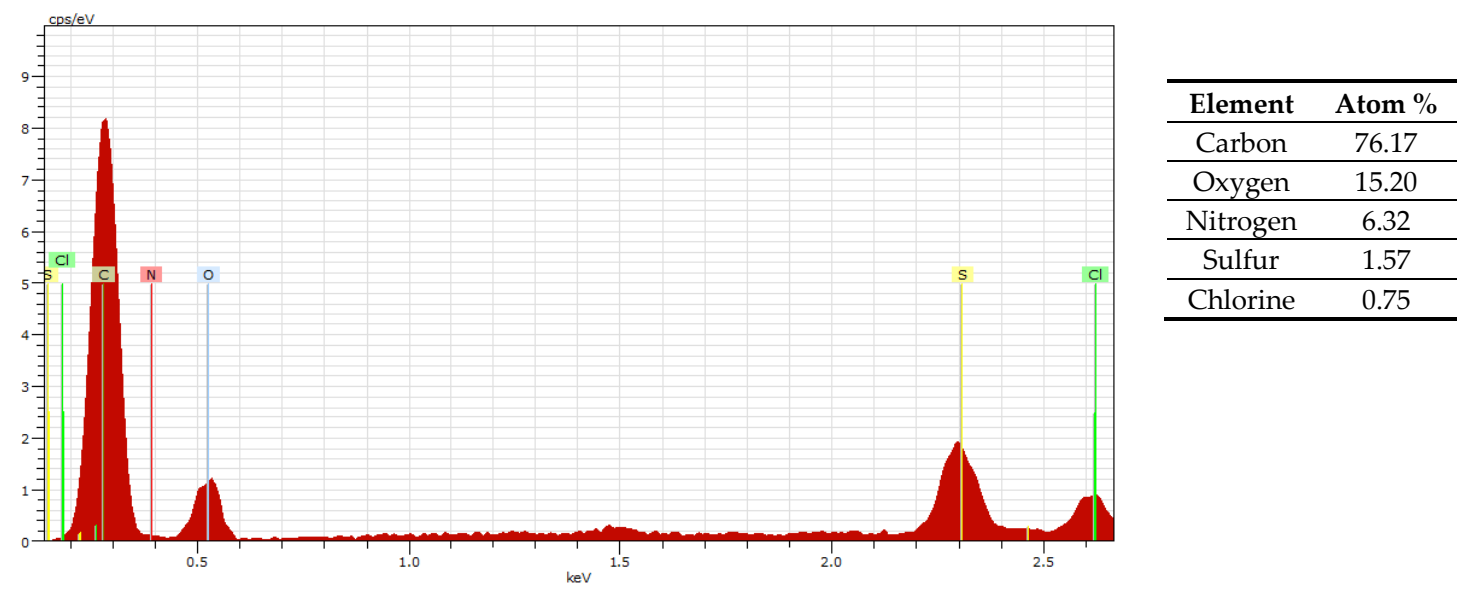

Figure 3. Energy Dispersive X-ray spectra and Atom Percent of C6-AGT.

\subsubsection{Fourier-Transformed Infrared Spectra}

Figures 4 and 5 show the FTIR spectra of SEGT and TosGT, respectively. Note that there is a significant decrease in the intensity of the $\mathrm{O}-\mathrm{H}$ band in the $3500-3000 \mathrm{~cm}^{-1}$ region. The presence of the tosylate group is substantiated by the peak at $1174 \mathrm{~cm}^{-1}$ assigned to symmetrical stretching and the asymmetrical stretching at $1364 \mathrm{~cm}^{-1}$ of the $\mathrm{S}=\mathrm{O}$ group in organic sulfonates. Various strong S-O-C stretching bands are seen in the $1000-769 \mathrm{~cm}^{-1}$ region, of which the bands at 817 and $667 \mathrm{~cm}^{-1}$ have been highlighted [14]. 


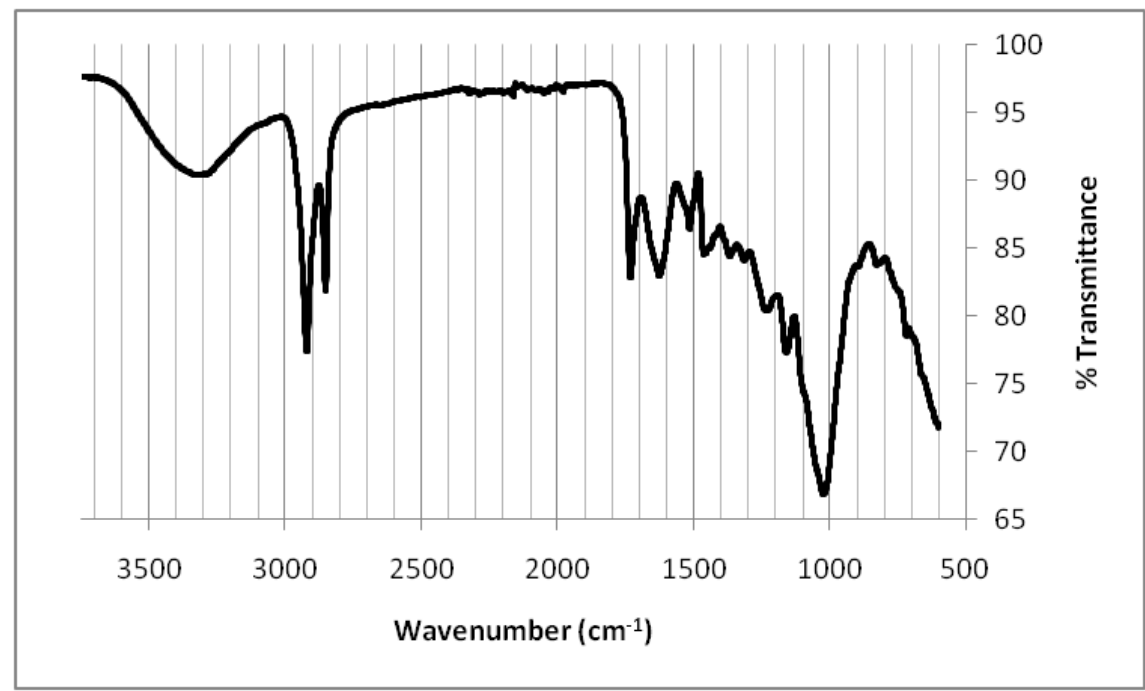

Figure 4. FTIR of SEGT (Soxhlet extracted green tea leaves).

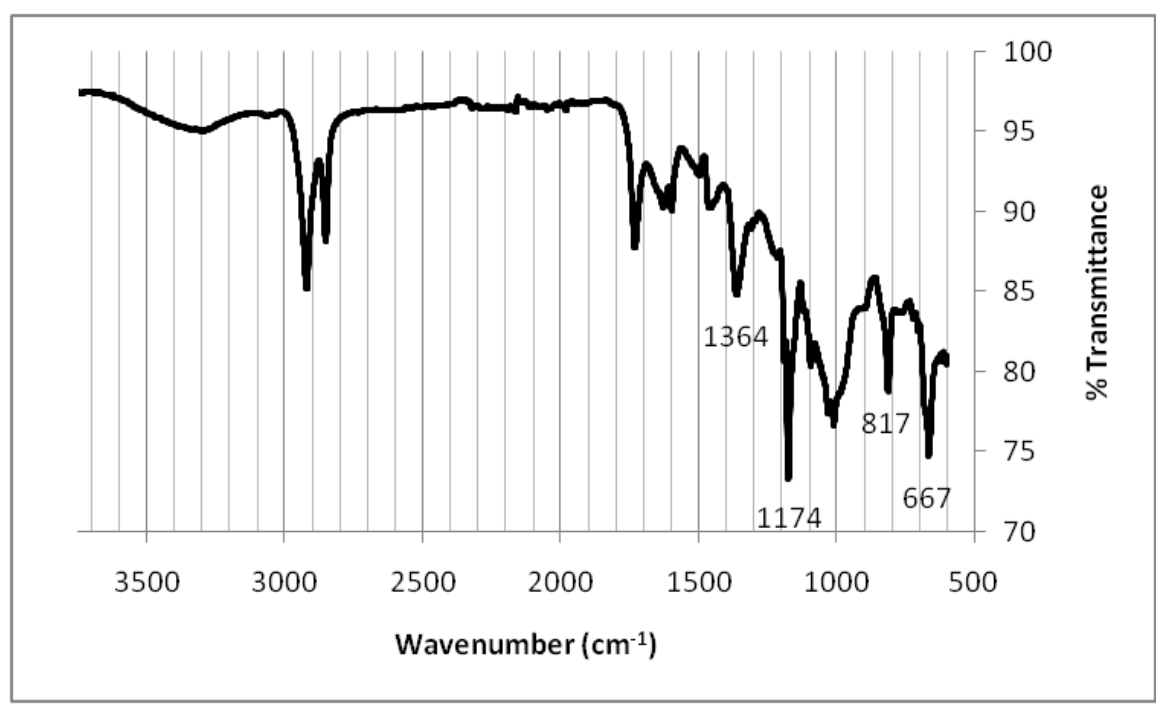

Figure 5. FTIR of the Tosylated SEGT.

The FTIR of the aminated (C6) tea leaves shown in Figure 6 does not appear that different from the tosylated tea leaves except for the shape around the $1000 \mathrm{~cm}^{-1}$ region. The band at $1019 \mathrm{~cm}^{-1}$ is most likely associated with the C-N stretch seen in aliphatic amines. On subtracting the spectrum of the tosylate from that of C6-AGT, an additional somewhat weaker band at $1225 \mathrm{~cm}^{-1}$ is seen. This is also attributed to $\mathrm{C}-\mathrm{N}$ vibrations from the amine functionality. Some of the other bands due to amines are broad and are most likely buried under the tosylate functionality that has not been fully displaced. The FTIRs of the $\mathrm{C} 8, \mathrm{C} 10$, and $\mathrm{C} 12$ aminated tea leaves are very similar to the IR of the C6 aminated tea leaves shown in Figure 6.

\subsubsection{Morphological Texture}

The microscopic view of the surfaces was obtained to determine whether the two chemical steps used in modifying the leaves created any significant morphological changes. These are presented in Figures 7-9. Note that the "rosette" structures seen in SEGT in Figure 7a are also seen on the surfaces of the four aminated tea leaves in Figure $8 \mathrm{a}, \mathrm{b}$ and Figure $9 \mathrm{a}, \mathrm{b}$. This suggests that the reactions performed 
on SEGT did not change the morphology. Since the physical integrities of the four aminated tea leaves appear to be similar, their ability to interact with 2-CP is most likely not due to textural differences.

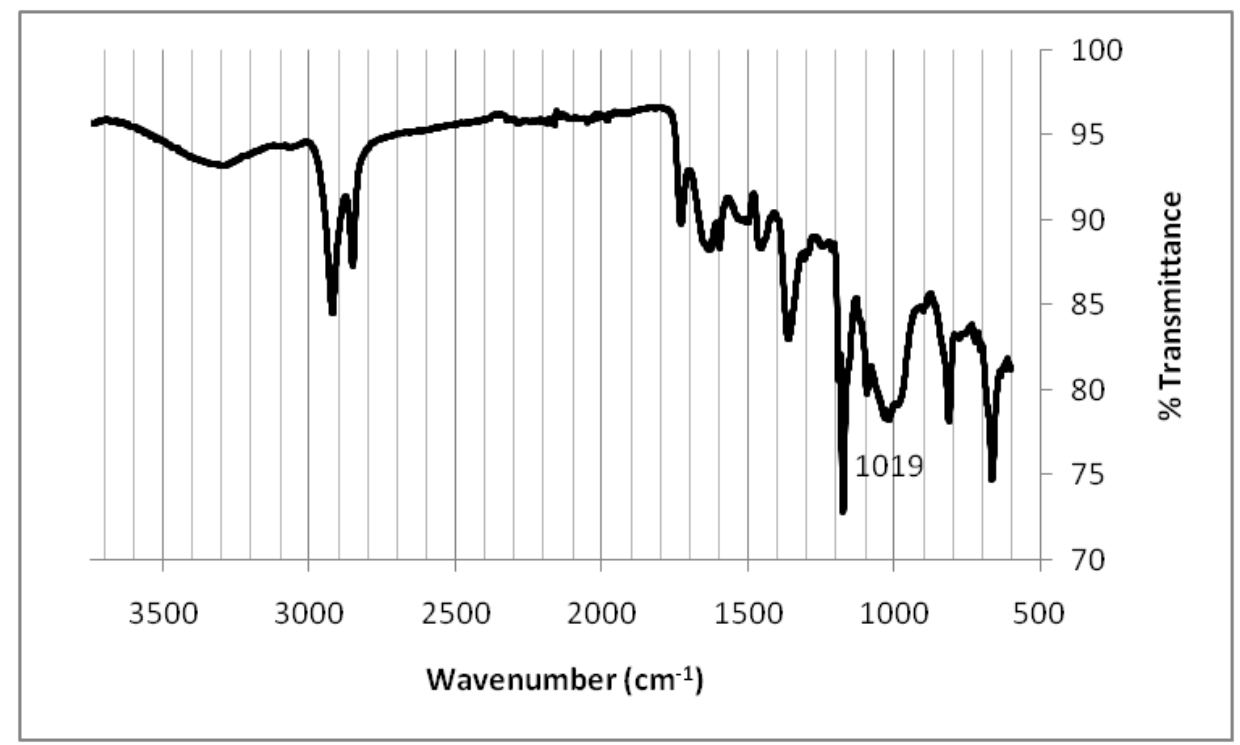

Figure 6. FTIR of the C6 aminated tea leaves.

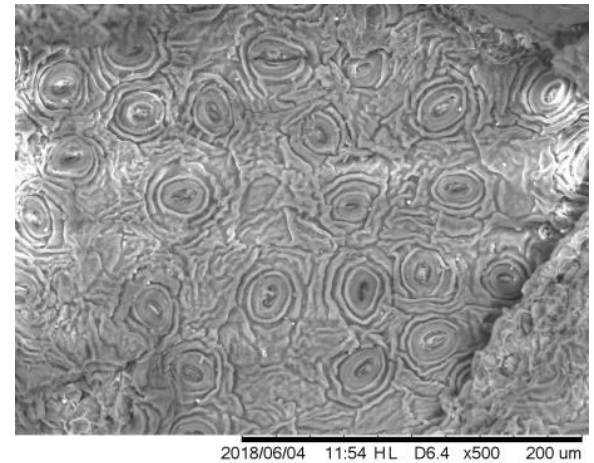

(a)

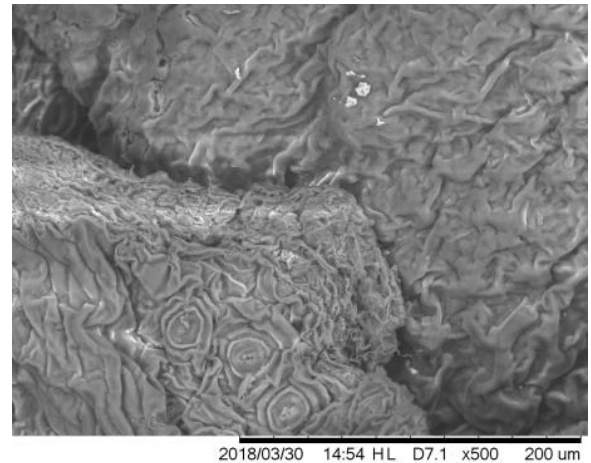

(b)

Figure 7. Scanning electron micrographs of (a) SEGT and (b) TosGT.

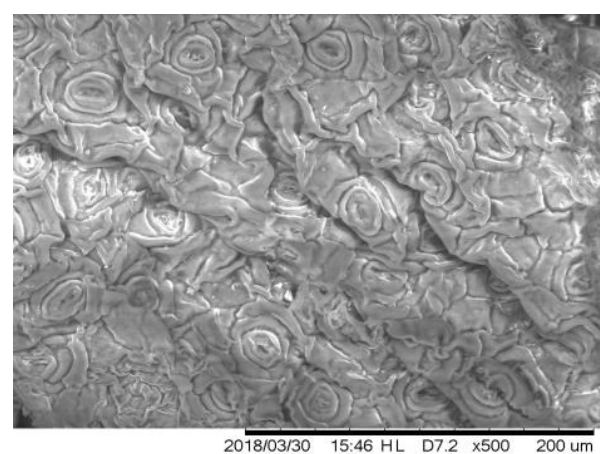

(a)

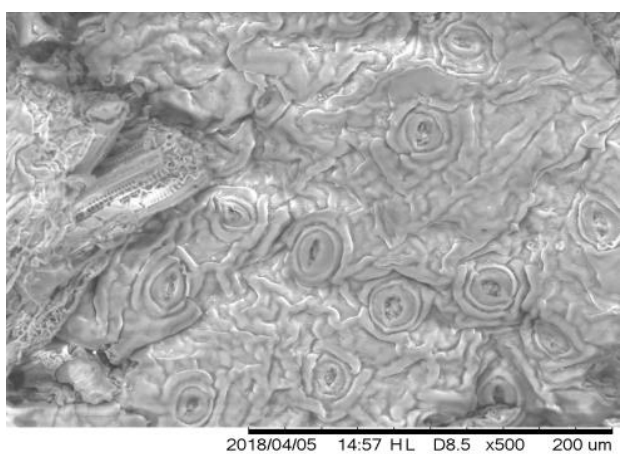

(b)

Figure 8. Scanning electron micrographs of (a) C6-AGT and (b) C8-AGT. 


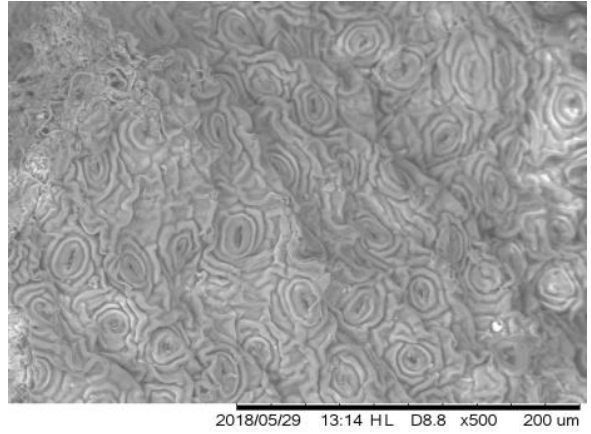

(a)

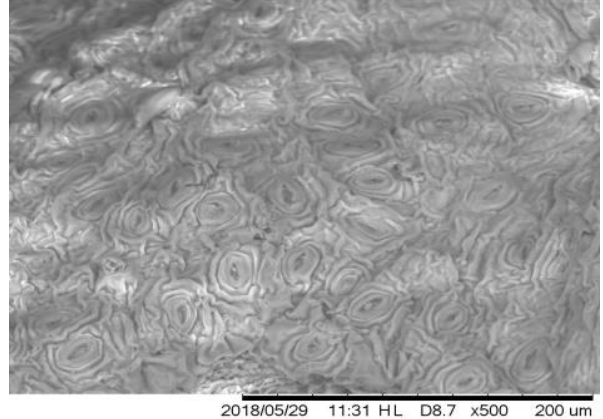

(b)

Figure 9. Scanning electron micrographs of (a) C10-AGT and (b) C12-AGT.

\subsubsection{Confirmation of Amine Functionality}

In the case of modifying a polycarbonate (PC) surface to introduce amine groups [15], neither XPS nor ATR-FT-IR spectroscopy definitively identified the presence of functional amines at the surface of the treated PC samples. Therefore, a surface-specific test to confirm the presence of primary amine groups was devised for their application.

As can be seen from the data presented thus far, the case for the aminated tea leaves parallels the aminated surface analysis of the polycarbonate. The dye assay for surface analysis for primary amines is based on a chemical reaction that uses either picric acid or acid orange 7 [16]. Rather than develop a dye-specific test for amines based on these two phenols, the following experiments with 2-CP provided evidence for the presence of the amine functionality.

Preliminary evidence for the presence of the amine group was obtained by adding the aminated and native tea leaves to varying concentrations of 2-CP solutions. After brief vortexing, the $\mathrm{pH}$ of the solution was gauged using litmus paper. The $\mathrm{pH}$ of a $2-\mathrm{CP}$ solution $(2 \%)$ rose from 4 to 6 at a $500 \mathrm{mg}$ loading of C6-AGT. On the other hand, even at loadings in the range of 940 to $1500 \mathrm{mg}$ of finely ground SEGT, there was no change in the $\mathrm{pH}$.

Further, aminated GTs have shown a significantly higher ability to pick up phenol compared to native tea leaves. This is shown in the results below. Also, the desorption studies discussed below confirm the presence of "basic" functional groups in the aminated tea leaves.

\subsection{Phenol Analysis}

\subsubsection{Effect of $\mathrm{pH}$}

An initial study using SEGT and C6-AGT was performed to determine whether the removal of 2-CP would be sensitive to the $\mathrm{pH}$ of the solution. Based on the study, it became clear that under acidic $\mathrm{pH}$ conditions the amine group on the surface of the modified tea leaves most likely exists as the ammonium ion $\mathrm{R}^{-\mathrm{NH}_{3}}{ }^{+}$, making it unable to interact with the acidic phenolic group in 2-CP. Under basic conditions, the phenolate ion of 2-CP would not be able to interact with the basic amine functionality in AGTs. This result corroborated with the work done by Bernal et al. [17]. Their study confirmed the phenol was adsorbed on the basic sites of activated carbon best at $\mathrm{pH}$ values close to neutral. The remaining two studies (effect of varying mass of adsorbent and concentration of 2-CP) were therefore performed using the 2-CP solution "as-such" without any $\mathrm{pH}$ adjustments.

\subsubsection{Effect of Varying the Mass of Material}

The concentration of the 2-CP solution used in this study was $50 \mathrm{ppm}$. The materials included in the study were activated charcoal (AC), SEGT, C6 AGT, C10-AGT, and C12-AGT. The amounts of adsorbent used were $50 \mathrm{mg}, 75 \mathrm{mg}, 100 \mathrm{mg}, 150 \mathrm{mg}$, and $200 \mathrm{mg}$. Each of the amounts was studied in duplicate for each material. Thus, 50 of the brown threaded tubes were labeled and charged with the 
appropriate amounts and type of adsorbent. The tubes were then filled with $30 \mathrm{~mL}$ of the 2-CP solution and allowed to equilibrate as described in Section 2.5. The solutions were then analyzed for 2-CP as described in Section 2.6. The percentages of 2-CP removed are based on the decrease in absorbance compared to that of the original solution $(50 \mathrm{ppm})$. The results thus obtained are summarized in Table 3. For each entry in this table, the mean percentage removal (over two analyses) is shown along with the mean absolute deviation in parenthesis.

Table 3. Percentages of 2-CP removed from a $50 \mathrm{ppm}$ solution using varying masses of adsorbent.

\begin{tabular}{cccccc}
\hline Material & $\mathbf{5 0} \mathbf{~} \mathbf{g}$ & $\mathbf{7 5} \mathbf{~} \mathbf{g}$ & $\mathbf{1 0 0} \mathbf{~} \mathbf{g}$ & $\mathbf{1 5 0} \mathbf{~} \mathbf{g}$ & $\mathbf{2 0 0} \mathbf{~ m g}$ \\
\hline SEGT & $5.3(2.2)$ & $4.8(0.6)$ & $6.0(0.5)$ & $8.7(0.3)$ & $12.0(0.8)$ \\
C6-AGT & $21.8(0.7)$ & $24.7(1.3)$ & $25.8(0.1)$ & $32.5(1.9)$ & $32.1(0.9)$ \\
C10-AGT & $20.7(0.7)$ & $21.1(0.8)$ & $24.2(0.5)$ & $26.7(0.2)$ & $27.6(0.6)$ \\
C12-AGT & $14.7(0.7)$ & $17.2(0.1)$ & $16.3(0.1)$ & $16.7(0.7)$ & $22.8(0.1)$ \\
AC & $89.3(0.9)$ & $89.3(0.2)$ & $89.4(0.9)$ & $89.2(0.8)$ & $90.5(0.7)$ \\
\hline \multicolumn{7}{c}{ AC, activated carbon. }
\end{tabular}

The goal of this study was to determine the mass of adsorbent beyond which the 2-CP removal levels off for this particular configuration used in the experiment. The configuration is defined by the following parameters: the dimensions of the tube and the volume of the solution used, temperature, and the orbital agitation used at the speed studied. As can be seen from Table 3, the ability of activated carbon to remove $2-\mathrm{CP}$ reached $90 \%$ even at $50 \mathrm{mg}$. The SEGT and the aminated tea leaves were used as such without creating fine particles. The C6-AGT removes 2-CP almost 3-4 times better than SEGT. Among the different AGTs, C6 appears to be the best. In the case of the C6-AGT, the removal capacity levels off at a loading of $150 \mathrm{mg}$ of the adsorbent. Therefore, in the next study, when the effect of varying concentrations of the $2-\mathrm{CP}$ solution was studied, the amount of adsorbent used was set to $150 \mathrm{mg}$.

\subsubsection{Effect of Varying the Concentration of 2-CP}

Since activated charcoal and SEGT were at the two ends in their ability to remove phenol, one much better, and the other significantly worse than the AGTs, this study only focused on comparing the four AGTs. The varying initial concentrations used for the study were $50 \mathrm{ppm}, 75 \mathrm{ppm}, 100 \mathrm{ppm}$, $150 \mathrm{ppm}$, and $200 \mathrm{ppm}$ solutions of aqueous 2-CP. Brown threaded tubes were loaded with $150 \pm 1 \mathrm{mg}$ of the material and each tube received $30 \mathrm{~mL}$ of each of the concentrations of 2-CP. Each of the concentrations was studied in duplicate for each material. The mean percentage of 2-CP removed for the varying concentrations is summarized in Table 4 . For each entry in this table, the mean percentage removal (over two analyses) is shown along with the mean absolute deviation in parenthesis.

Table 4. Percentages of 2-CP removed using $150 \mathrm{mg}$ of adsorbent at varying concentrations of 2-CP.

\begin{tabular}{cccccc}
\hline Material & $\mathbf{5 0} \mathbf{p p m}$ & $\mathbf{7 5} \mathbf{p p m}$ & $\mathbf{1 0 0} \mathbf{p p m}$ & $\mathbf{1 5 0} \mathbf{p p m}$ & $\mathbf{2 0 0} \mathbf{p p m}$ \\
\hline C6-AGT & $32.9(0.1)$ & $41.4(2.3)$ & $38.3(0.2)$ & $40.3(0.7)$ & $38.9(0.7)$ \\
C8-AGT & $23.3(0.8)$ & $30.2(0.4)$ & $31.4(1.0)$ & $31.9(0.6)$ & $32.0(0.3)$ \\
C10-AGT & $26.6(1.1)$ & $35.7(0.1)$ & $36.8(0.5)$ & $39.6(0.7)$ & $41.7(0.4)$ \\
C12-AGT & $23.4(1.0)$ & $29.1(0.5)$ & $29.6(0.4)$ & $33.0(0.1)$ & $33.2(0.4)$ \\
\hline
\end{tabular}

Based on these studies, it has been concluded that tethering the amine group at the end of six atoms from the substrate produces the best results. Forty percent $(40 \%)$ of the 2 -CP is removed from aqueous solutions at a concentration of $75 \mathrm{ppm}$ when $150 \mathrm{mg}$ of the adsorbent is used.

The synthesis of C6-AGT has not been optimized. These results are therefore very encouraging because there is considerable scope for improvement. After having studied phenol removal, attention was focused on the desorption ability of these adsorbents. 


\subsubsection{Desorption Studies}

To release the phenol easily so that it can be re-used in the industry is just as important as being able to remove the phenol from the aqueous waste streams. 2-CP was expected to be removed by the amine groups in AGT via a reaction of the amine and phenol to form the ammonium phenolate entity. Therefore, using acid or base to release the sorbed phenol was expected to work quite well. In Section 2.7.1, the experiment performed for removing 2-CP from water using activated charcoal and C6-AGT is described. The adsorbent with the 2-CP was then desorbed using $0.1 \mathrm{~N} \mathrm{HCl}, 0.1 \mathrm{~N}$ aqueous $\mathrm{NaOH}$ solution, and DI water. Refer to Section 2.7.2 for the experimental details. Percentages of phenol adsorbed and desorbed were measured according to the details described in Section 2.6. The results obtained from these desorption studies are summarized in Table 5 . Note that the percentages are based on the amount of 2-CP that was adsorbed.

Table 5. Percentages of 2-CP desorbed using aq. acid, aq. base, and neutral water.

\begin{tabular}{ccc}
\hline Solution & C6-AGT & Activated Charcoal \\
\hline $0.1 \mathrm{~N} \mathrm{HCl}$ & 55.5 & 4.5 \\
$0.1 \mathrm{~N} \mathrm{NaOH}$ & 70.3 & 9.9 \\
DI Water & 27.0 & 3.4 \\
\hline \multicolumn{3}{c}{ DI, de-ionized. }
\end{tabular}

The reason for the higher amounts of phenol released with the base, for charcoal and the aminated tea, is most likely due to the higher solubility of the sodium phenoxide in water. On the other hand, under acidic conditions, 2-CP, which has a lower solubility in water compared to its sodium salt, is released. This is shown in Scheme 4.
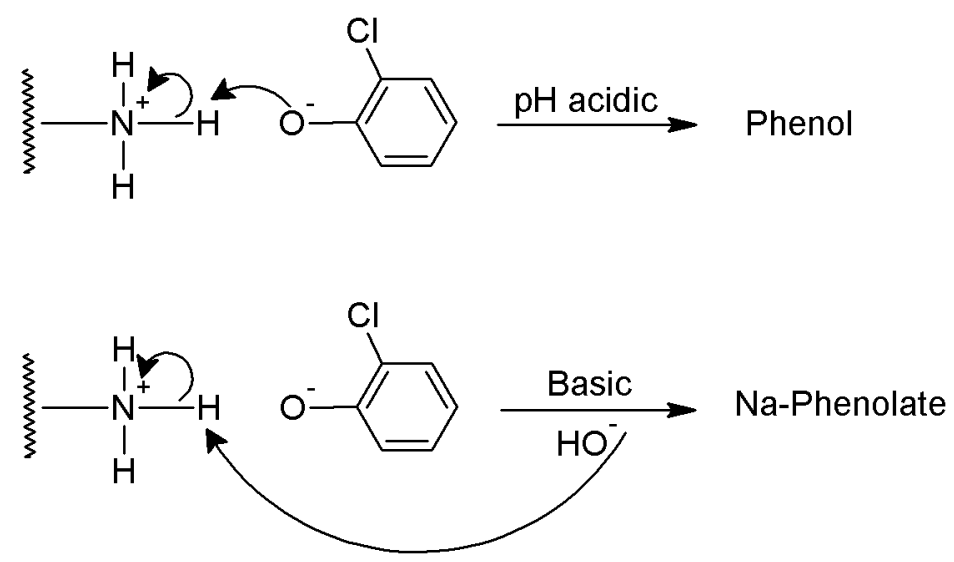

Scheme 4. Showing the $\mathrm{pH}$ dependence on Phenol release.

The more important point to be realized from the results in Table 5 is the much lower desorption in the case of the activated charcoal. This is most likely due to the 2-CP volatilizing off during the drying of the adsorbent to get rid of the water. The 2-CP is adsorbed on to the surface of the charcoal is most likely held in place by very weak physical forces, such as London dispersion forces. On the other hand, in the case of C6-AGT, the 2-CP is held in place by an acid-base reaction. Thus, the 2-CP could not volatilize off. It was most likely released by the reactions shown in Scheme 4.

In addition to the acid-base interactions, other weaker intermolecular forces also come into play in the case of C6-AGT. This is evident by noting that DI water was able to release a significant amount of the 2-CP from C6-AGT. This is most likely due to the substances in the lignin cellulosic portions of C6-AGT. In other words, the amine groups in C6-AGT interact with 2-CP via ionic interactions while the remaining biomass holds the 2-CP via $\mathrm{H}$-bonding and dipole-dipole interactions. 
Thus, the desorption studies clearly indicate that though charcoal removes a higher percentage of the 2-CP from aqueous solutions, it releases the phenol just as easily. Its method of removal is dependent on many "weaker" forces mainly because of its enhanced surface area. However, these weaker forces are "unzipped" more easily. The ability of the activated charcoal to give up the 2-CP so easily suggests that engineering controls would be needed in the case of activated charcoal.

The amination step has not been optimized. Use of higher temperatures in the amination step needs to be explored. It also needs to be seen if one of the amine groups in the diamine can be converted to the ammonium group as shown by Abel et al. [9]. This will solve the cross-linking issue by the introduction of a protection-deprotection step.

Thus, aminated tea leaves with an enhanced number of amine functional groups on the surface could offer the distinct advantage of easy and quantitative release of 2-CP compared to activated charcoal.

\section{Conclusions}

In this study, among the four aminated tea leaves, C6-AGT, which has a chain of six methylenes, showed the best adsorption capacity. About $40 \%$ of the 2-CP is removed from a 75 ppm aqueous solution at a loading of $150 \mathrm{mg}$. The percentages adsorbed and desorbed can be further improved by optimizing the reaction conditions used for making these aminated tea leaves.

Desorption using aqueous acidic or basic solutions released more than twice as much of the 2-CP compared to neutral water, strongly suggesting acid-base interactions. This confirmed our expectations that the amine group in the adsorbent would interact with the phenolic group in 2-CP via an acid-base reaction. Tethering the amine beyond six carbons does not appear to produce any additional benefit.

The ultimate goal of this project is to prepare this C6-AGT using aqueous environmentally friendly reagents. The issues with this approach have been well-documented in Melo et al.'s review of chemical modifications of lignocellulosics [18]. It appears that the green approaches lead to fewer reactions sites on the surface. Future work will explore the use of spacers of around six atoms to ameliorate this issue. The ultimate goal is to use bio-waste modified in an environmentally friendly manner to remove pollutants from our current environment.

Author Contributions: R.I. designed the synthetic schemes and supervised the project. M.F.-B. and S.T. worked on the synthetic experiments. J.Y. contributed to the quantitative experiments. A.E.N. developed the methodologies for phenol analysis and material characterization. R.I. and A.E.N. contributed to the writing and editing of the manuscript. All authors have read and approved the final manuscript.

Funding: This research was funded by a PSC-CUNY Award \# 60295-00 48 to R.I. The Program for Research Initiatives in Science and Math (PRISM) at John Jay College through the US Department of Education Title-V grant \# P031C110174 supported M.F.-B. via stipends and also helped with the purchase of chemicals and analyses. The CSTEP project provided funding for S.T. and J.Y.

Acknowledgments: R.I. would like to acknowledge K. Grohmann, Emeritus Professor, Hunter College, CUNY for discussions related to syntheses and Chiu Hong Lee for her technical support. Research Director Bach at BMCC and the Science Department at BMCC also played a key role in supporting this work. We would like to thank the anonymous reviewers for their critical reading of the manuscript and helpful comments.

Conflicts of Interest: The authors declare no conflict of interest.

\section{References}

1. Volesky, B. Sorption and Biosorption; BV Sorbex: Montreal, QC, Canada, 2003.

2. Saka, C.; Sahin, O.; Kucuk, M.M. Applications on agricultural and forest waste adsorbents for the removal of lead (II) from contaminated waters. Int. J. Environ. Sci. Technol. 2012, 9, 379-394. [CrossRef]

3. Desta, M.B. Batch Sorption Experiments: Langmuir and Freundlich Isotherm Studies for the Adsorption of Textile Metal Ions onto Teff Straw (Eragrostis tef) Agricultural Waste. J. Thermodyn. 2013, 2013, 375830. [CrossRef] 
4. Yildiz, S. Kinetic and Isotherm Analysis of $\mathrm{Cu}(\mathrm{II})$ Adsorption onto Almond Shell (Prunus Dulcis). Ecol. Chem. Eng. S 2017, 24, 87-106. [CrossRef]

5. Deng, S.; Bai, R. Behaviors and mechanisms of copper adsorption on hydrolyzed polyacrylonitrile fibers. J. Colloid Interface Sci. 2003, 260, 265-272. [CrossRef]

6. Zhang, Y.; Gan, T.; Luo, Y.; Zhao, X.; Hu, H.; Huang, Z.; Huang, A.; Qin, X. A green and efficient method for preparing acetylated cassava stillage residue and the production of all-plant fibre composites. Compos. Sci. Technol. 2014, 102, 139-144. [CrossRef]

7. Padil, V.; Wacławek, S.; Černík, M. Green Synthesis: Nanoparticles and Nanofibres Based on Tree Gums for Environmental Applications. Ecol. Chem. Eng. S 2016, 23, 533-557. [CrossRef]

8. Ramdani, D.; Chaudhry, A.S.; Seal, C.J. Chemical composition, plant secondary metabolites, and minerals of green and black teas and the effect of different tea-to-water ratios during their extraction on the composition of their spent leaves as potential additives for ruminants. J. Agric. Food Chem. 2013, 61, 4961-4967. [CrossRef] [PubMed]

9. Abel, T.; Cohen, J.L.I.; Engel, R.; Filshtinskaya, M.; Melkonian, A.; Melkonian, K. Preparation and investigation of antibacterial carbohydrate-based surfaces. Carbohydr. Res. 2002, 337, 2495-2499. [CrossRef]

10. Zhang, W.-B.; Li, Y.; Li, X.; Dong, X.; Yu, X.; Wang, C.-L.; Wesdemiotis, C.; Quirk, R.P.; Cheng, S.Z.D. Synthesis of Shape Amphiphiles Based on Functional Polyhedral Oligomeric Silsesquioxane End-Capped Poly(L-Lactide) with Diverse Head Surface Chemistry. Macromolecules 2011, 44, 2589-2596. [CrossRef]

11. Miyafuji, H. Application of ionic liquids for effective use of woody biomass. J. Wood Sci. 2015, 61, 343-350. [CrossRef]

12. Eaton, A.D.; Clesceri, L.S.; Rice, E.W.; Greenberg, A.E.; Franson, A.A.H. Standard Methods for the Examination of Water and Wastewater, 21st ed.; American Public Health Association: Washington, DC, USA, 2005; pp. 5-47. ISBN 978-0875530475.

13. Hueser, E.; Heath, M.; Schockley, W.H. The Rate of Esterification of Primary and Secondary Hydroxyls of Cellulose with p-Toluenesulfonyl (Tosyl) Chloride. J. Am. Chem. Soc. 1950, 72, 670-674. [CrossRef]

14. Silverstein, R.M.; Bassler, G.C.; Morrill, T.C. Spectrophotometric Identification of Organic Compounds, 4th ed.; Wiley: New York, NY, USA, 1981; ISBN 0-471-02990-4.

15. Yeow, B.; Coffey, J.W.; Muller, D.A.; Grøndahl, L.; Kendall, M.A.F.; Corrie, S.R. Surface Modification and Characterization of Polycarbonate Microdevices for Capture of Circulating Biomarkers, Both In Vitro and In Vivo. Anal. Chem. 2013, 85, 10196-10204. [CrossRef] [PubMed]

16. Goddard, J.M.; Hotchkiss, J.H. Polymer surface modification for the attachment of bioactive compounds. Prog. Polym. Sci. 2007, 32, 698-725. [CrossRef]

17. Bernal, V.; Erto, A.; Giraldo, L.; Moreno-Piraján, J.-C. Effect of Solution pH on the Adsorption of Paracetamol on Chemically Modified Activated Carbons. Molecules 2017, 22, 1032. [CrossRef] [PubMed]

18. Melo, D.Q.; Neto, V.D.O.S.; Barros, F.C.D.F.; Raulino, G.S.C.; Vidal, C.B.; Nascimento, R.F.D. Chemical modifications of lignocellulosic materials and their application for removal of cations and anions from aqueous solutions. J. Appl. Polym. Sci. 2016, 133, 43286. [CrossRef]

(C) 2018 by the authors. Licensee MDPI, Basel, Switzerland. This article is an open access article distributed under the terms and conditions of the Creative Commons Attribution (CC BY) license (http:// creativecommons.org/licenses/by/4.0/). 